\title{
Significance of Ki-67 in Prognostication of Soft Tissue Tumors
}

\author{
Sridevi. $V^{1 *}$, Susruthan Muralitharan ${ }^{2}$, and Thanka. $J_{.}^{2}$ \\ 'Pathology, Sri Muthukumaran Medical college and Hospital, India \\ ${ }^{2}$ Pathology, Sri Ramachandra medical college and Research institute, India
}

\begin{abstract}
Background: Histological grading is the most important prognostic factors and the best indicator of metastatic risk in adult soft tissue sarcomas. It has been shown that Ki67 immunohistochemical (IHC) staining is an effective method of assessing the prognosis in a number of tumor types. Ki67 is a cell cycle antigen which is elevated in proliferation states. High level of Ki67 expression is an independent prognostic indicator that correlates with poor outcome in patients with sarcomas.

Methods: In this study 53 cases of soft tissue tumor were selected and were classified according to FNCLCC system. IHC staining of Ki-67 was done in all the 53 samples. Diagnosis was made mainly based on histopathological pattern analysis and with the use of IHC.

Results: By using FNCLCC grading soft tissue tumor cases were graded as grade I, II and III. The correlation between the FNCLCC grading and Ki67 was done and it was observed that there was statistically significant positive correlation between Ki67 and the grade of the tumor, as evidenced by P- values of 0.002, 0.005 and 0.004. The correlation between the FNCLCC grading and Ki67 index was also assessed. Statistically significant positive correlation between Ki67 index and the grade of the tumor was observed.

Conclusion: There was significant correlation noted between FNCLCC grading and Ki-67 index. Thus it can be recommended that Ki-67 IHC stain should be done on routine basis to accurately grade the sarcomas so that it will be beneficial for the management of the patient.
\end{abstract}

Keywords: FNCLCC Grading, Immunohistochemical Staining, Ki-67 Index, Soft Tissue Tumors

\section{Introduction}

Soft tissue tumors are composed of numerous and complex diagnostic entities. Because of this complexity and the recognition of an intermediate malignancy category including some tumors with a deceptive bland histological appearance, soft tissue tumors represent a major diagnostic challenge to the general practicing pathologist. Despite the rapid development of molecular genetic technique, immunohistochemistry plays an important role in the diagnosis of soft tissue tumors.

The histological type of sarcomas does not always provide sufficient information for predicting the clinical course and therefore for planning therapy. The two most widely used systems are the NCI (United States National Cancer Institute) system ${ }^{[1,2]}$ and the FNCLCC (French Federation Nationale des Centres de Lutte Contre le Cancer) system. ${ }^{[3-7]}$

The FNCLCC system is based on a score obtained by evaluating three parameters selected after multivariate analysis of several histological features: tumour differentiation, mitotic rate and amount of tumour necrosis.

${ }^{[7]} \mathrm{A}$ score is attributed independently to each parameter and the grade $(\mathrm{G})$ is obtained by adding the three attributed scores. Tumour differentiation is highly dependent on histological type and subtype. ${ }^{[7]}$

Histological grading is the most important prognostic factors and the best indicator of metastatic risk in adult soft tissue sarcomas. Sarcomas are also characterized by deregulated proliferation. Ki67 is a cell cycle antigen which is elevated in proliferation states. High level of Ki67 expression is an independent prognostic indicator that correlates with poor outcome in patients with sarcomas.

Current classification schemes may require revision where biological behavior and prognostic significance of these tumors is concerned, as an increasing number of studies have suggested that Ki67 may be an important factor in cancer grading and prognostic evaluation. It has been shown that Ki67 immunohistochemical (IHC) staining is an effective method of assessing the prognosis in a number of tumor types. ${ }^{[8,9]}$

Although pKi67 is a key marker associated with proliferating cancer cells and a poor prognosis, its full potential in increasing proliferation has not been evaluated. In syngeneic animal models with subcutaneous or orthotopic bladder cancer, prostate cancer or renal cell carcinoma, antisense oligonucleotides induced tumor 
growth inhibition ${ }^{[10,11]}$, potentially through the inhibition of $\mathrm{Ki}-67$, indicating the involvement of $\mathrm{Ki} 67$ in tumor cell proliferation.

\section{Materials and Methods}

The present study was conducted in the department of Pathology of Sri Ramachandra Medical College and Research Institute. All soft tissue tumor diagnosed between January 2006 to June 2011 were retrieved from the surgical pathology files. A total of 513 cases were collected and reviewed. Out these cases benign, intermediate and malignant cases were identified. The malignant cases were taken out of which small biopsy specimens and patients who lacked details of important pathological features were excluded from the study.

All the soft tissue tumor specimens which were surgically resected and subsequently diagnosed as one of malignant soft tissue tumor by histopathological examination with Hematoxylin and Eosin (H\&E) stain were included in the study.

The clinical features such as age, sex of the patient and location of the tumor were collected. The gross characteristics of the tumor which included the tumor location, size, necrosis, circumscription, cut section and secondary changes were obtained from the pathology report registers. Paraffin blocks of concerned cases were recovered and histological sections (5 to $6 \mathrm{um}$ ) were routinely stained with $\mathrm{H} \& \mathrm{E}$ stains.

H\&E stained sections were reviewed along with the grading of the tumours according to FNCLCC grading system. The microscopic features were assessed after reviewing all the available slides. These included the histological pattern, cellular features, pleomorphism, mitosis, vascularity and secondary changes. Diagnosis was made mainly based on histopatholgical pattern analysis and with the use of Ki67 analysis.

For doing Ki67 staining 53 cases of sarcomas were selected. Representative slides were selected after looking for the areas of maximum mitosis and absence of necrosis.
Immunostaining of Ki-67 antigen was done using Ki-67 monoclonal antibody (antihuman Ki-67 antigen, AM297$5 \mathrm{M}$, Biogenex, at a dilution of 1:50). Sections from lymph node were included as positive control. Negative control (without adding primary antibody) was included in all the batches. Sections were examined under high power field to observe the immunoreactivity. Hot spot (area with highest density of immunostained nuclei ) was selected and adjacent fields counted to include 1000 nuclei. Distinct nuclear staining of the tumor cells was recorded as positive. Ki-67 Labeling Index (LI) was recorded as percentage of positively stained tumor nuclei in 1000 tumor cells. Vascular components and inflammatory cells were excluded. Necrotic, degenerated and poorly preserved areas were also excluded.

\section{Result}

FNCLCC grading was used to grade the study cases and it was observed that $35.8 \%$ of soft tissue tumours cases were of grade I, $26.4 \%$ were of grade II and $37.7 \%$ were of grade III. This table (Table no:1) shows the percentage of various grades of sarcomas which are included in the study of 53 cases. The percentage of grade III sarcomas (37.7\%) is more compared with other grades.

The correlation between the FNCLCC grading and Ki67 was done. It was observed that there was statistically significant positive correlation between $\mathrm{Ki} 67$ and the grade of the tumor, as evidenced by $\mathrm{P}$ - values of 0.002 , 0.005 and 0.004 (Table no:2). The mean of ki-67 index in different grades were assessed (figure:1) and the mean of ki-67 for 1000 nuclei in different grades were also assessed (figure:2) which showed that ki index is comparatively more in grade III sarcomas with percentage of 10.86 . The grade I and grade II shows $1.32 \% \& 6.33 \%$.

The overall comparison of values between H\&E mitosis per $10 \mathrm{HPF}$ with Ki67/ $10 \mathrm{HPF}$ showed significant correlation with $\mathrm{P}$ value of 0.0005 (Table:3). The correlation between the FNCLCC grading and Ki67 index was also assessed. Statistically significant positive correlation between Ki67 index and the grade of the tumors was observed (Table no:4).

Table No.1: Distribution of tumor according to FNCLCC grading.

\begin{tabular}{|l|c|c|}
\hline FNCLCC Grade & N & $\%$ \\
\hline I & 19 & 35.8 \\
\hline II & 14 & 26.4 \\
\hline III & 20 & 37.7 \\
\hline Total & $\mathbf{5 3}$ & $\mathbf{1 0 0}$ \\
\hline
\end{tabular}


Table No. 2: Comparison of FNCLCC grading and Ki67 index.

\begin{tabular}{|c|c|c|c|c|c|c|}
\hline \multirow{2}{*}{ FNCLCC grading } & \multirow{2}{*}{$\mathbf{N}$} & \multirow{2}{*}{ Mean } & \multirow{2}{*}{ SD } & \multirow{2}{*}{ SE } & \multicolumn{2}{|c|}{$95 \%$ confidence interval for mean } \\
\hline & & & & & Lower bound & Upper bound \\
\hline I & 19 & 20.15 & 23.36 & 5.36 & 8.89 & 31.42 \\
\hline II & 14 & 63.28 & 50.38 & 13.46 & 34.19 & 92.37 \\
\hline III & 20 & 108.6 & 54.78 & 12.25 & 82.96 & 134.23 \\
\hline Total & 53 & 64.92 & 58.19 & 7.99 & 48.88 & 80.96 \\
\hline
\end{tabular}

\begin{tabular}{|l|c|c|c|c|c|}
\hline Dependent variable & FNCLCC grading (i) & FNCLCC grading (j) & mean difference (i-j) & Std Error & Significance \\
\hline \multirow{3}{*}{ Ki 1000} & \multirow{2}{*}{ I } & II & -43.13 & 15.73 & 0.008 \\
\cline { 2 - 6 } & \multirow{2}{*}{ II } & III & -88.44 & 14.31 & 0.000 \\
\cline { 2 - 6 } & \multirow{2}{*}{ III } & I & 43.13 & 15.73 & 0.008 \\
\cline { 2 - 6 } & & III & -45.31 & 15.57 & 0.005 \\
\cline { 2 - 6 } & & II & 48.44 & 14.31 & 0.000 \\
\hline
\end{tabular}

Table No. 3 :Comparison between H\&E mitosis/ 10 hpf and Ki 67/10 hpf.

\begin{tabular}{|l|c|c|}
\hline \multirow{2}{*}{$\mathrm{H} \&$ E mitosis/ 10 HPF } & \multicolumn{2}{|c|}{ Ki/ 10 HPF } \\
\cline { 2 - 3 } & No & P value \\
\cline { 2 - 3 } & 53 & 0.0005 \\
\hline
\end{tabular}

Table No. 4: Comparison of FNCLCC grading and Ki67 index.

\begin{tabular}{|c|c|c|c|c|c|c|}
\hline \multirow{2}{*}{$\begin{array}{l}\text { FNCLCC } \\
\text { grading }\end{array}$} & \multirow{2}{*}{$\mathbf{N}$} & \multirow{2}{*}{ Ki Index Mean } & \multirow{2}{*}{ SD } & \multirow{2}{*}{ SE } & \multicolumn{2}{|c|}{$95 \%$ confidence interval for mean } \\
\hline & & & & & Lower bound & Upper bound \\
\hline I & 19 & 1.31 & 1.56 & 0.36 & 0.57 & 2.07 \\
\hline II & 14 & 6.33 & 5.04 & 1.35 & 3.42 & 9.24 \\
\hline III & 20 & 10.86 & 5.48 & 1.22 & 8.29 & 13.42 \\
\hline Total & 53 & 6.24 & 5.93 & 0.81 & 4.61 & 7.89 \\
\hline
\end{tabular}

\begin{tabular}{|l|c|c|c|c|c|}
\hline Dependent variable & FNCLCC grading & FNCLCC grading & Mean difference & Std Error & Significance \\
\hline \multirow{3}{*}{ Ki Index } & \multirow{3}{*}{ I } & II & -5.01 & 1.53 & 0.002 \\
\cline { 2 - 5 } & \multirow{3}{*}{ II } & III & -9.53 & 1.39 & 0.000 \\
\cline { 2 - 6 } & \multirow{3}{*}{ III } & I & 5.01 & 1.53 & 0.002 \\
\cline { 2 - 6 } & & III & -4.53 & 1.51 & 0.004 \\
\cline { 2 - 6 } & & II & 9.53 & 1.39 & 0.000 \\
\hline
\end{tabular}

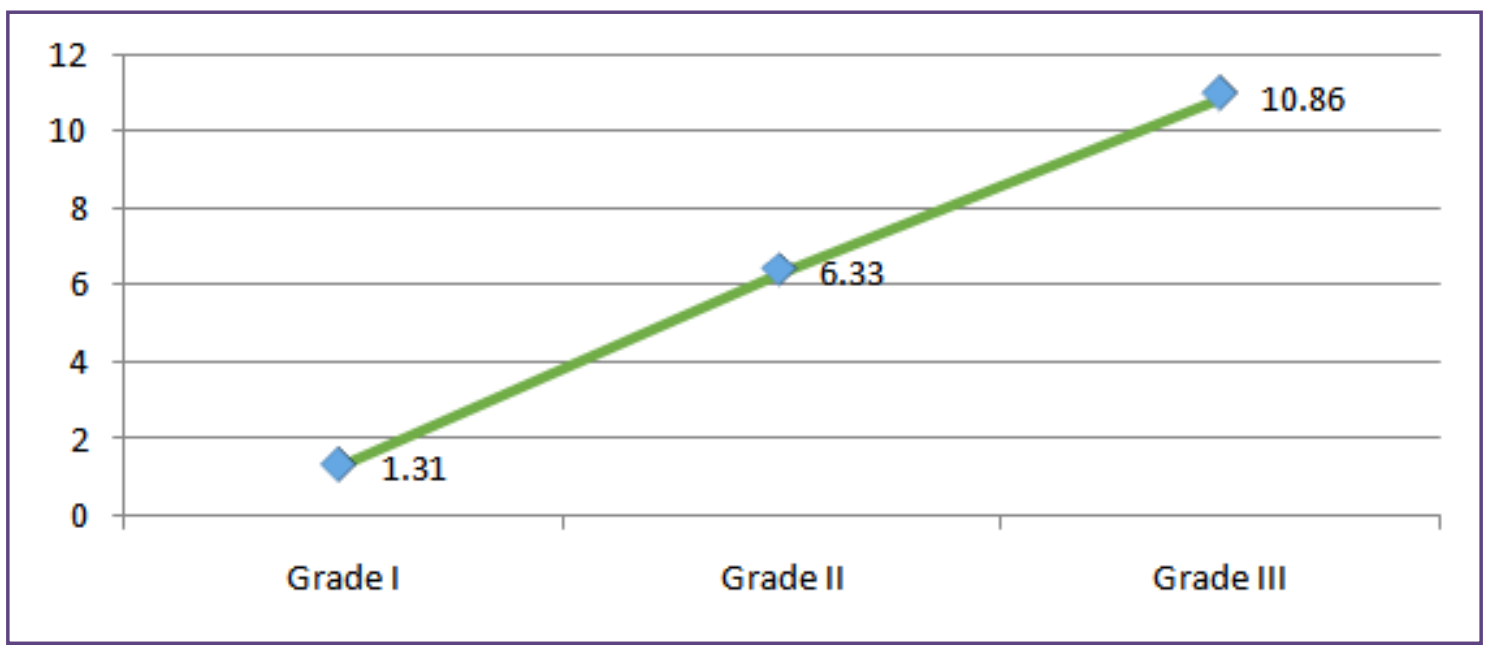

Fig.1: Comparison of FNCLCC grading and Ki67 index. 


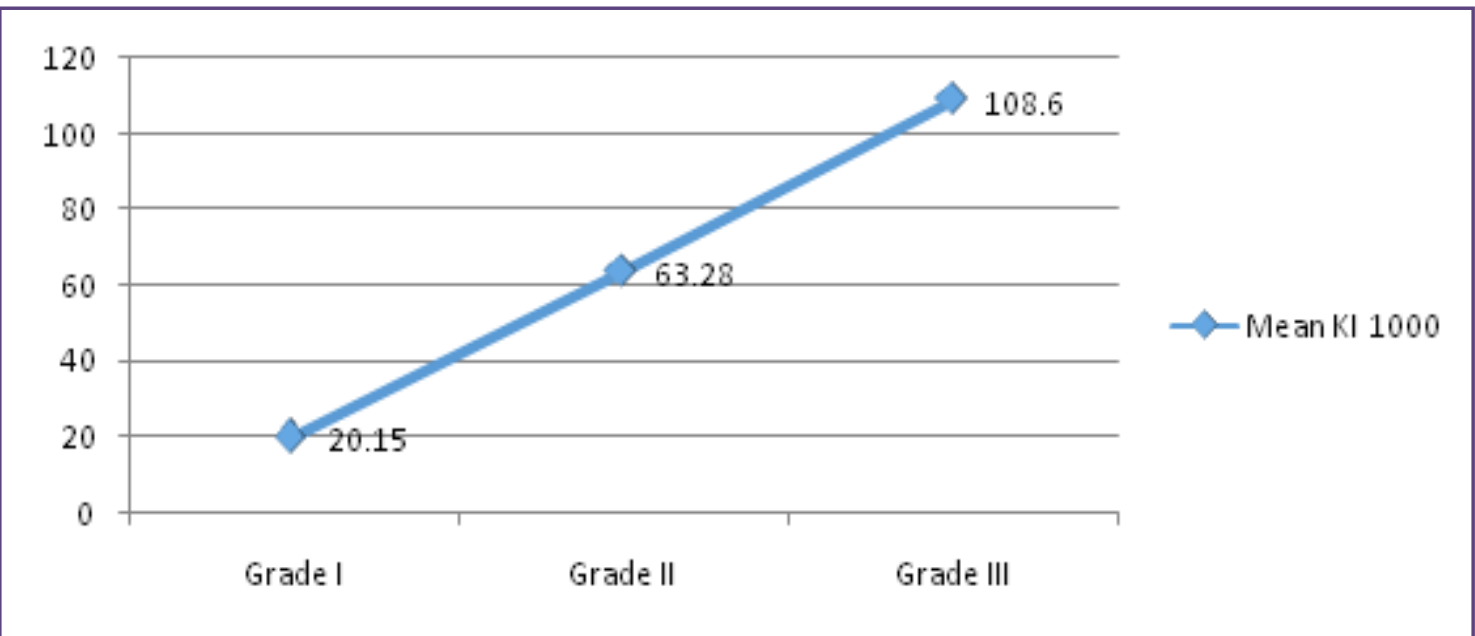

Fig. 2: Comparison of FNCLCC grading and Ki67 score for 1000 nuclei.

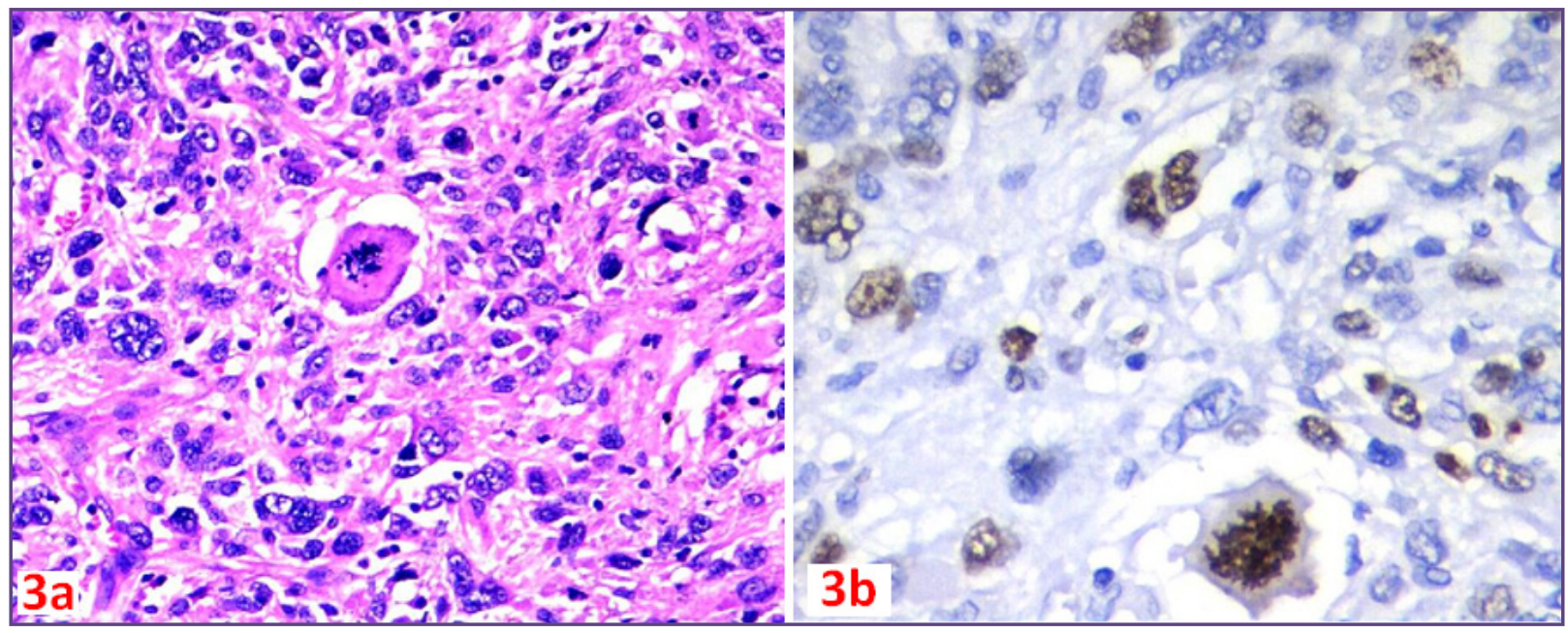

Fig. 3a: H\&E 40X- pleomorphic sarcoma sarcoma(G III): shows highly pleomorphic cells with atypical mitosis. Fig:3b: 40XKi67 immunostain: mitosis highlighted.

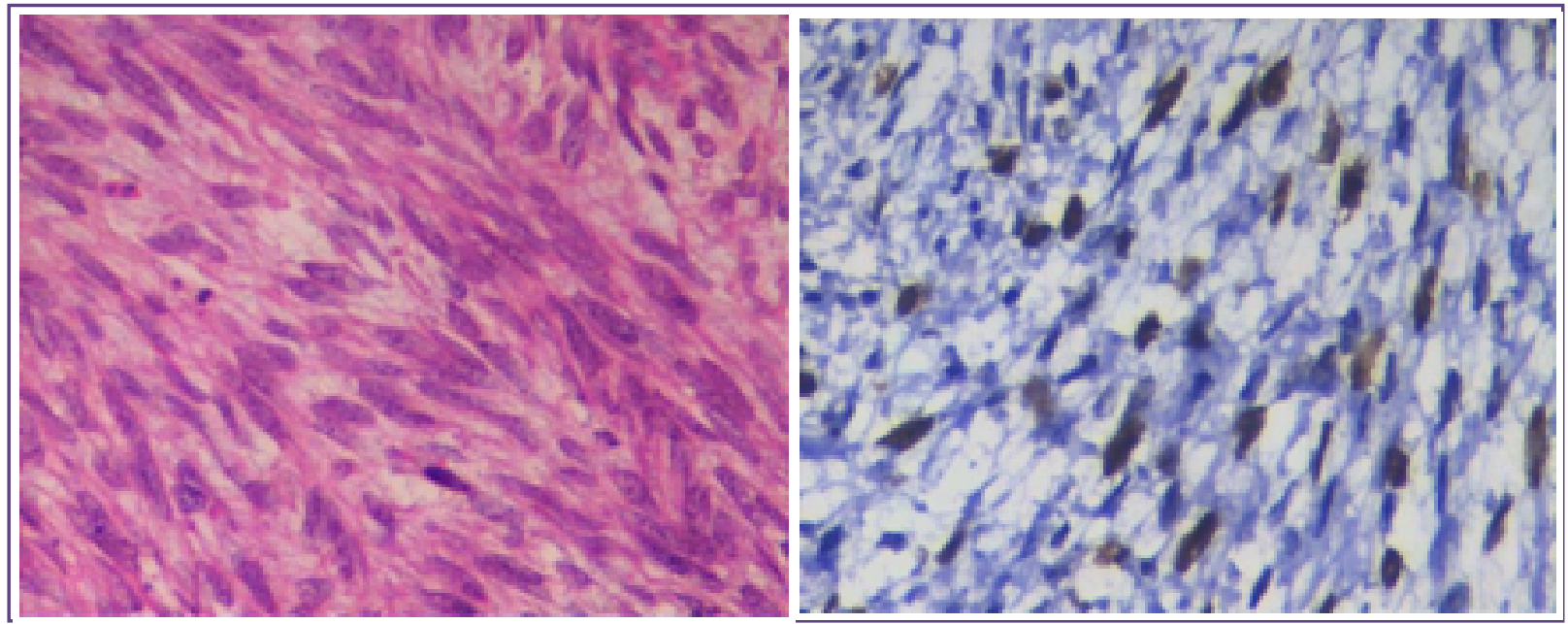

Fig. 4a:H\&E 40X-fibrosarcoma (GII): spindle shaped cells arranged in herringbone pattern. Fig:4b: 40X Ki67 immunostain. 


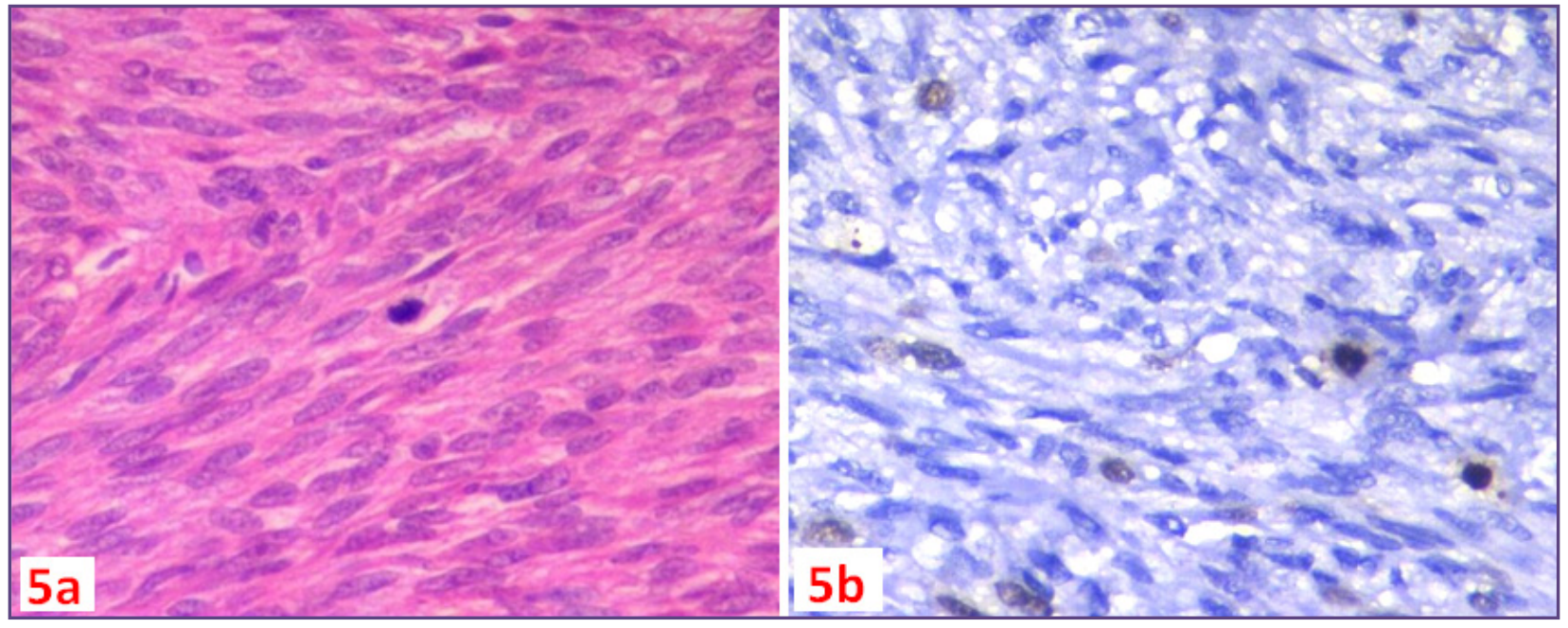

Fig. 5a: H\&E 40X- DFSP(GI) shows spindle shaped cells. Fig5b: 40X Ki67immunostainhighlights mitosis.

\section{Discussion}

The present study was conducted to analyze the importance of Ki67 index in soft tissue tumours and to see the correlation between FNCLCC grading and Ki67analysis in 53 cases of sarcomas. According to FNCLCC grading it was observed that $35.8 \%$ of soft tissue tumours cases were of grade I, $26.4 \%$ were of grade II and $37.7 \%$ were of grade III.

Immunohistochemistry is the most valuable adjuvant to $\mathrm{H} \& \mathrm{E}$ staining in diagnostic histopathology. It is important in diagnosis of STT because of their variety with several lines of differentiation and the frequent difficulty of diagnosis with numerous pseudosarcomatous benign lesion and non mesenchymal tumors.

$\mathrm{Ki} 67$ is frequently used as an indicator of cell proliferation. [12,13] Ki67 was significantly more highly expressed in malignant than in normal tissues. ${ }^{[14,15]}$ The nuclear proliferative antigen $\mathrm{Ki} 67$ is identified to correlate with the prognosis of the patients to predict the outcome and distant metastasis of sarcoma patients. A significant correlation has been reported between Ki67 expression and mitotic rate in sarcomas. ${ }^{[16,17]}$ A correlation between ki67 reactivity and tumour grade in sarcomas has been detected in different retrospective studies.

Ki67 IHC staining was done on 53 cases of various sarcomas which had different grades. The correlation between the FNCLCC grading and Ki67 index was assessed. Statistically significant positive correlation between Ki67 index and the grade of the tumor was observed(Fig 3-5). Sahil et al have reported Ki67 index to be very low in benign and G-I tumour and high in G-II and G-III tumor. Sarcomas in our study with G-III also had high Ki index compared to GI tumours which correlated with the study.
The Ki67\% was less than $10 \%$ in G-I sarcomas in our study which correlated well with Aydin etal study which also showed Ki67 $\%<10 \%$ in all of the G-I tumours (100\%). In his study all the G-III tumours had Ki67\% more than $10 \%$. But in our study few of the G-III sarcomas like Leiomyosarcoma, MFH, synovial sarcoma had $<10 \%$ which did not correlate with Aydin et al study. But it correlated with the study of Swanson et al. They have also observed a disparity between Ki67\% and histologic grade in some tumor. The Ki67 index strongly correlated with various histological grade with $p$ value of 0.002 with G-I and G-II, 0.0005 in G-I and G-III, 0.004 in G-II and G-III sarcomas.

Comparing Ki67 for $10 \mathrm{HPF}$ and mitotic index which was used in FNCLCC grading showed that more number of mitosis is counted with $\mathrm{Ki} 67$ than routine mitotic index using H\&E stain. This will help to increase the grade of the sarcomas perfectly and the treatment is also improved. Takafumi veda et al showed positive correlation between Ki67 per $10 \mathrm{HPF}$ and number of mitotic figure per $10 \mathrm{HPF}$. The present study also showed positive correlation with $\mathrm{P}$ value of 0.0005 between Ki67 per $10 \mathrm{HPF}$ and number of mitotic figure per $10 \mathrm{HPF}$.

The findings in present study indicate that precise assessment of the proliferative index using Ki-67 immunohistochemical stain can be a valuable adjunct to routine histopathology and in treatment of patients. The data on Ki67 as a diagnostic marker is scarce and based on varying laboratory and statistical methods. Cancer has a complex pathogenesis and reliable early diagnosis is difficult. ${ }^{[18]}$ Symptoms usually do not appear until the disease has progressed to an advanced stage. Therefore, further research into diagnostic and prognostic markers may aid early diagnosis. 


\section{Conclusion}

Thus we conclude that there was significant correlation noted between FNCLCC grading and Ki-67 Index. Thus it can be recommended that $\mathrm{Ki}-67 \mathrm{IHC}$ stain should be done on routine basis to accurately grade the sarcomas so that it will be beneficial for the management of the patient.

\section{References}

1. Sharon W. Weiss and John R. Goldblum. Enzigner \& Weiss's Soft Tissue Tumors, 5th edition; 2008.

2. Jean-Michel Coindre et al. Grading of Soft Tissue Sarcomas. Review and Update. Arch Pathol Lab Med. October 2006 ;130: 1448-1453.

3. Coindre JM, Terrier P, Bui NB, Bonichon F, Collin F, Le D, V, Mandard AM, et al. Prognostic factors in adult patients with locally controlled soft tissue sarcoma. A study of 546 patients from the French Federation of Cancer Centers Sarcoma Group. J Clin Oncol. 1996; 14: 869-877.

4. Coindre JM, Terrier P, Guillou L, Le D, V, Collin F, Ranchere $\mathrm{D}$, et al. Predictive value of grade for metastasis development in the main histologic types of adult soft tissue sarcomas: a study of 1240 patients from the French Federation of Cancer Centers Sarcoma Group. Cancer. 2001; 91: 1914-1926

5. Neuville A et al.Grading of soft tissue sarcomas: from histological to molecular assessment. The journal of royal college of pathologist of Australia. 2014; 46( 2): 113-120.

6. Oliveira AM, Nascimento AG.Grading in soft tissue tumors: principles and problems. Springer-Verlag. October 2001; 30(10): 543-559.

7. Guillou L, Coindre JM, Bonichon F, Nguyen BB, Terrier P, Collin F, et al. Comparative study of the National Cancer Institute and French Federation of Cancer Centers Sarcoma Group grading systems in a population of 410 adult patients with soft tissue sarcoma. J Clin Oncol. 1997; 15: 350-362.

8. Scholzen T, Gerdes J. The Ki-67 protein: from the known and the unknown. J Cell Physiol2000; 182:311-22.
9. Jacquemier JD, Penault Llorca FM, Bertucci F, et al: Angiogenesis as a prognostic marker in breast carcinoma with conventional adjuvant chemotherapy: a multiparametric and immunohistochemical analysis. J Pathol. 1998; 184: 130-135.

10. Kausch I, Lingnau A, Endl E, et al: Antisense treatment against Ki-67 mRNA inhibits proliferation and tumor growth in vitro and in vivo. Int J Cancer. 2003; 105: 710-716.

11. Liu J, Fang L, Cheng Q, et al: Effects of G250 promoter controlled conditionally replicative adenovirus expressing Ki67-siRNA on renal cancer cell. Cancer Sci. 2012; 103: 1880-1888.

12. Karamitopoulou E, Perentes E, Tolnay M and Probst A: Prognostic significance of MIB1, p53, and bcl2 immunoreactivity in meningiomas. Hum Pathol.1998; 29: 140-145.

13. Geyer FC, Rodrigues DN, Weigelt B and Reis Filho JS: Molecular classification of estrogen receptor positive/ luminal breast cancers. Adv Anat Pathol. 2012; 19: 39-53.

14. Claudio PP, Zamparelli A, Garcia FU, et al: Expression of Cell Cycle regulated Proteins pRb2/p130, p107, p27kip1, p53, mdm2, and Ki67 (MIB1) in Prostatic Gland Adenocarcinoma1.Clin Cancer. 2002; 8:1808-15.

15. HY Hu, Liu H, JW Zhang, et al: Clinical significance of Smac and Ki-67 expression in pancreatic cancer. Hepatogastroenterology.2012; 59: 2640-2643.

16. Golouh R, Bracko M, Novak J. Predictive value of proliferation related markers p53 and DNA ploidy for survival in patients with soft tissue spindle cell sarcomas. Mod. Pathol. 1996; 9: 919-924.

17. Hoos A, Stojadinovic A, Mastorides S et al. High Ki-67 Proliferative Index Predicts Disease Specific Survival in Patients with High- Risk Soft Tissue Sarcomas. Cancer 2001; 92: 869-74.

18. Lian Tao Li, Guan Jiang, Qian Chen, Jun Nian Zheng. Ki67 is a promising molecular target in the diagnosis of cancer (Review). Molecular Medicine Reports. 2011; 11: 1566-1572.

*Corresponding author:

Sridevi.V, 52(Old No:39), Gangadeeshwaran Koil Street, Nutech-Sreenivas Apartment, Flat:C-9, Purasaiwalkam, Chennai:600084(India)

Phone: +919444952854

Email: drsridevi78@yahoo.co.in

Date of Submission : 16.06.2017

Date of Acceptance : 09.07.2017

Financial or other Competing Interests: None.

Date of Publication : 30.10.2017 